\title{
Detection of serum human epididymis secretory protein 4 in patients with ovarian cancer using a label-free biosensor based on localized surface plasmon resonance
}

\author{
This article was published in the following Dove Press journal: \\ International Journal of Nanomedicine \\ 12 June 2012 \\ Number of times this article has been viewed
}

\author{
Jialing Yuan' \\ Ruiqi Duan' \\ Huan Yang ${ }^{2}$ \\ Xiangang Luo ${ }^{2}$ \\ Mingrong $\mathrm{Xi}^{\prime}$ \\ 'Department of Gynecology and \\ Obstetrics, West China Second \\ University Hospital, Sichuan \\ University, ${ }^{2}$ State Key Laboratory \\ of Optical Technologies for \\ Microfabrication, Institute of Optics \\ and Electronics, Chinese Academy \\ of Science, Chengdu, China
}

\begin{abstract}
Background: Detection of the human epididymis secretory protein 4 (HE4) biomarker plays an important role in the early diagnosis of ovarian cancer. This study aimed to develop a novel localized surface plasmon resonance (LSPR) biosensor for detecting HE4 in blood samples from patients with ovarian cancer.

Methods: Silver nanoparticles were fabricated using a nanosphere lithography method. The anti-HE4 antibody as a probe, which can distinctly recognize HE4, was assembled onto the nanochip surface using an amine coupling method. Detection was based on the shift in the extinction maximum of the LSPR spectrum before and after the HE4-anti-HE4 antibody reaction. These nanobiosensors were applied to detect HE4 in human serum samples and compare them using an enzyme-linked immunosorbent assay.
\end{abstract}

Results: Tests relating to the detection of HE4 demonstrated that the LSPR-based biosensor featured a fast detection speed, good specificity, effective reproducibility, and long-term stability. The linear range for LSPR was between $10 \mathrm{pM}$ and 10,000 pM, with a detection limit of $4 \mathrm{pM}$. An excellent correlation between LSPR and enzyme-linked immunosorbent assay results was observed in human serum.

Conclusion: This study is the first clinical diagnostic application of the LSPR biosensor in ovarian cancer. The LSPR biosensor, a rapid, low-cost, label-free and portable screening tool, can serve as a very effective alternative for the clinical serological diagnosis of ovarian cancer.

Keywords: localized surface plasmon resonance system, nanobiosensor, ovarian cancer biomarker, serum HE4 protein

\section{Introduction}

Since the advent of nanotechnology, nanoscale particle-based sensors have attracted tremendous attention from scientists, because of their unique optical and electrical properties. ${ }^{1-4}$ Localized surface plasmon resonance (LSPR), which is recognized as one of the special optical properties of noble metallic nanoparticles (eg, silver or gold), is generated when the incident photon frequency is resonant with the collective oscillation of conduction electrons. ${ }^{5}$ The LSPR biosensor, a novel type of optical fiberbased biosensor, uses an optical fiber or optical fiber bundle to transform biological recognition information into analytically useful signals in the LSPR spectrum, and has been proven to be an effective platform for detection techniques. ${ }^{5,6}$ The sensing principle is based on its sensitivity to local refractive index changes near the nanoparticle surface induced by biomolecular interactions. ${ }^{5-8}$ The applicability of this nanobiosensor has 
been studied in many fields, such as drug screening, medical diagnostics, and environmental monitoring, and has become a hot research topic all over the world. ${ }^{9-13}$

The detection of biotin-streptavidin and microalbumin in patients' urine using the proposed domestic LSPR biosensor has been reported previously, without quantitative analysis. ${ }^{14,15}$ However, to date, this biosensor has not been widely utilized in the field of gynecological oncology.

Ovarian cancer is one of the most common malignancies of the female reproductive system. According to the American Cancer Society, ovarian cancer accounts for about $4 \%$ of cancers occurring in women, but ranks fourth among the cancer-related deaths in women, because most cases are unfortunately diagnosed at an advanced stage. ${ }^{16}$ Currently, CA125 is the only biomarker of ovarian cancer that is most widely and routinely used in clinical practice. However, the clinical use of CA125 as a marker for early detection is severely restricted, because it is elevated in only half of early-stage ovarian cancers and is elevated frequently in many benign gynecological diseases, such as endometriosis, ovarian cysts, and pelvic inflammation. ${ }^{17,18}$ Recently, the human epididymis secretory protein 4 (HE4), which is a novel biomarker for ovarian cancer, has been widely studied and used in the early diagnosis of ovarian cancer. Reportedly, HE4 is highly sensitive to early ovarian cancer and can be used in combination with CA125, offering the best method of differential diagnosis in ovarian cancer and other pelvic masses. ${ }^{19-21}$

Currently used approaches for detection of HE4 are the enzyme-linked immunosorbent assay (ELISA) and the chemiluminescent immunoassay (CLIA). Although it is one of the most mature methods for protein detection used in the last three decades and is considered the gold standard, ELISA still has certain shortcomings in terms of the long assay time required, the indirect detection format, and the need for multiple washing steps. ${ }^{22}$ CLIA also has some disadvantages, including the large volume of the analysis instrument, high cost, and special labeling requirements. These factors constrain the clinical application of these techniques. Thus, a rapid, label-free, simple, low-cost, and portable protocol for detecting HE4 is urgently required.

In the present work, the LSPR biosensor developed was utilized based on silver nanoparticles for the direct detection of the HE4 biomarker in blood samples from patients with ovarian cancer. Under the optimum conditions, HE4 in both buffer and human serum samples are tested. The results are then compared using ELISA.
Based on current information, this study is the first to investigate the LSPR system for the detection of HE4. The study is also the first to discuss and analyze in detail the detection limit, linear range, and regeneration of the proposed homemade LSPR sensor.

\section{Materials and methods \\ Materials}

11-Mercaptoundecanoic acid and bovine serum albumin were purchased from Sigma-Aldrich (St Louis, MO). N-hydroxysuccinimide and 1-ethyl-3-(3-dimethylaminopropyl) carbodiimide hydrochloride (EDC) were purchased from Aladdin (Shanghai, China). Mouse monoclonal anti-HE4 antibody (anti-HE4) and standard HE4 were obtained from Abnova (Taiwan, China). Ethanolamine was purchased from the Kelang Company (Chengdu, China). An HE4 ELISA kit was obtained from Fujirebio Diagnostics Inc (Malvern, PA). Ultrapure water $(18.3 \mathrm{M} \Omega / \mathrm{cm})$ used for the preparation of all solutions was obtained from Millipore Co (Boston, MA). All reagents used were of analytical reagent grade.

\section{Patients and samples}

The human serum specimens were collected from West China Second University Hospital (Chengdu, China). Written informed consents were not obtained, because these samples were from leftover blood samples in routine blood tests, and this study did not cause any harm to the patients. Sera were isolated through the centrifugation of whole blood samples at $2000 \mathrm{rpm}$ for 20 minutes, and subsequently kept frozen at $-80^{\circ} \mathrm{C}$ until analysis.

\section{Preparation of LSPR biosensor and experimental setup}

The integrated LSPR biosensor used in this work was a custom system built on-site, as previously described in detail. ${ }^{14,23}$ The silver nanochip was fabricated using the nanosphere lithography method. The peak wavelength of the LSPR extinction spectrum $\left(\lambda_{\max }\right)$ excited by the silver nanoparticles was measured and recorded using an ultraviolet-visible spectroscope (Sciencetech 9055, Sciencetech, Ottawa, Canada) with a charge-coupled device detector (Koan Electro-Optics Co, Shanghai, China). ${ }^{14}$

The entire measurement process could be described as follows. The white light emerging from an optical fiber bundle and the transmitted light coupled into the detection probe of the optical fiber bundle provided the incident light. The nanochip was placed perpendicular to the incident light, which was taken using the ultraviolet-visible spectrometer 
ranging from $400 \mathrm{~nm}$ to $800 \mathrm{~nm}$ at room temperature in air. ${ }^{14}$ All the extinction spectra could be calculated through a software program (Ocean Optics, Dunedin, FL) and directly displayed on the screen of the computer. Thus, the data of different nanochips could be compared. The shift toward longer wavelengths, defined as a red shift, was indicated as $(+)$, whereas the shift toward shorter wavelengths, defined as a blue shift, was indicated as (-). The relative wavelength shift, namely $\Delta \lambda_{\max }$, was used to monitor the binding of target analytes. ${ }^{13}$ The experimental setup is illustrated in Figure 1.

\section{Functionalization of LSPR biosensor}

Functionalization is a multistep process that prepares the LSPR nanosensor for biodetection events (Figure 2). First, the silver nanochip was incubated in $1 \mathrm{mM} 11$-mercaptoundecanoic acid solution (in ethanol) for 12 hours to form a selfassembled monolayer on the slice surface, after which the nanochip was washed thoroughly with ethanol and dried at room temperature. The nanochip was then immersed in $75 \mathrm{mM}$ EDC/15 mM N-hydroxysuccinimide solution for 2 hours to activate the carboxyl groups of the self-assembled monolayer, which reacts with amino groups of antibodies to form amides. Subsequently, $50 \mu \mathrm{L}$ of anti-HE4 solution at $10 \mu \mathrm{g} / \mathrm{mL}$ was spotted on the self-assembled monolayermodified surface and overnight incubation at $4{ }^{\circ} \mathrm{C}$ followed. The anti-HE4 immobilized surface was immersed in $1 \mathrm{M}$ ethanolamine solution ( $\mathrm{pH} \mathrm{8.5)} \mathrm{for} 30$ minutes to deactivate the unreacted esters, after which the surface was washed with phosphate-buffered solution (pH 7.4) and dried. Finally, an HE4 nanobiosensor was formed.

\section{Detection of HE4 by LSPR and ELISA}

In the detection stage, the different concentrations of standard HE4 $(1 \mathrm{pM}$ to $0.1 \mu \mathrm{M})$ and the patient samples were incubated on the functionalized LSPR chip for 40 minutes, followed by a thorough rinsing with phosphate-buffered solution containing $0.05 \%$ Tween-20 to dissociate the nonspecific binding. Quantitative determination was performed using the ELISA kit according to the manufacturer's instructions. Each value was averaged from three parallel experiments. A statistical evaluation of the correlation of the LSPR and ELISA methods was performed and computed using a software program (Origin 8.0, OriginLab Corporation, Northampton, MA).

\section{Results and discussion LSPR response to immobilization process}

The LSPR spectra of the nanobiosensor in each processing step are shown in Figure 3. Before modification, the LSPR $\lambda_{\max }$ of the bare silver nanochip was measured at $592.58 \mathrm{~nm}$ (Figure 3A). A representative LSPR $\lambda_{\text {max }}$ of the silver nanochip after modification with 11-mercaptoundecanoic acid was $619.85 \mathrm{~nm}$ with a corresponding LSPR $\Delta \lambda_{\text {max }}$ of $+27.27 \mathrm{~nm}$ (Figure 3B). After anti-HE4 immobilization, the LSPR $\lambda_{\text {max }}$ shifted to $630.97 \mathrm{~nm}$, with an additional $11.12 \mathrm{~nm}$ red shift (Figure 3C). After incubation in $500 \mathrm{pM}$ HE4, the LSPR wavelength shifted to $+14.48 \mathrm{~nm}$, showing a $\lambda_{\max }$ of $645.45 \mathrm{~nm}$ (Figure 3D). This experimental evidence clearly showed that HE4 in the buffer solution was detected successfully by the LSPR biosensor. The optical characteristics of the nanosensor are notably based on the wavelength shift of the absorbance peak, $\Delta \lambda_{\max }$.

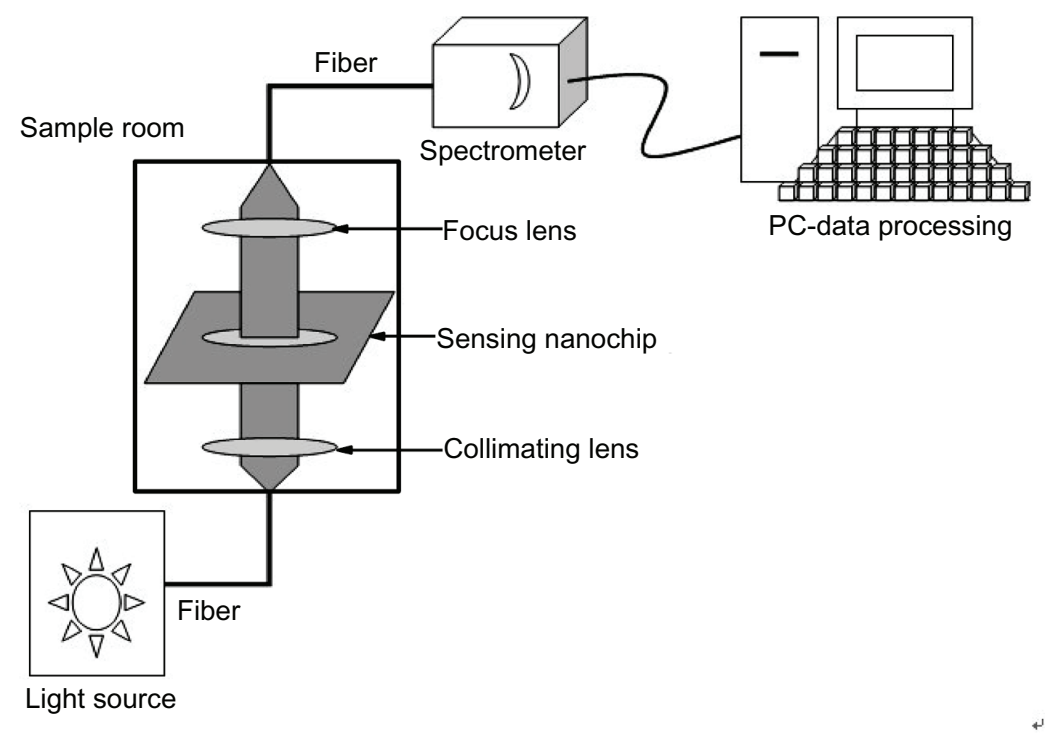

Figure I Schematic presentation of localized surface plasmon resonance experimental setup. 


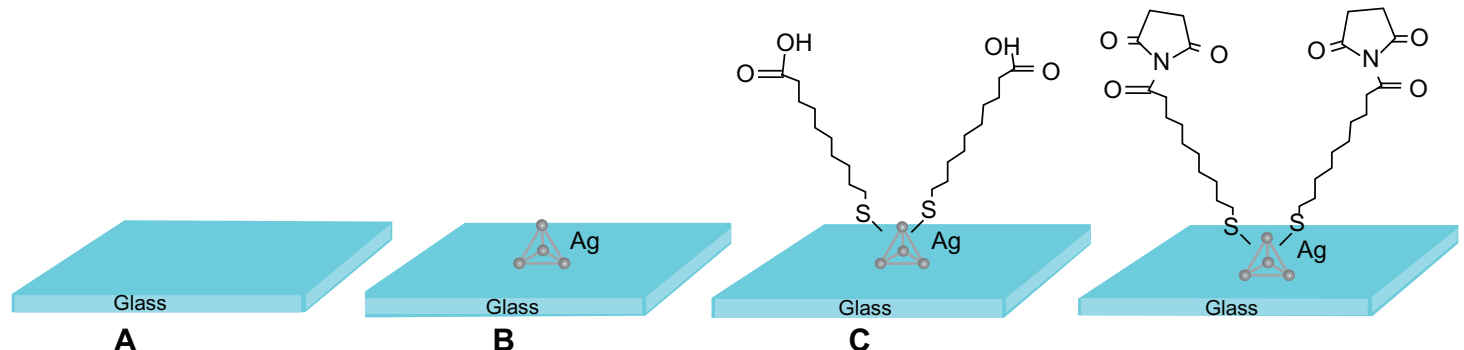

A
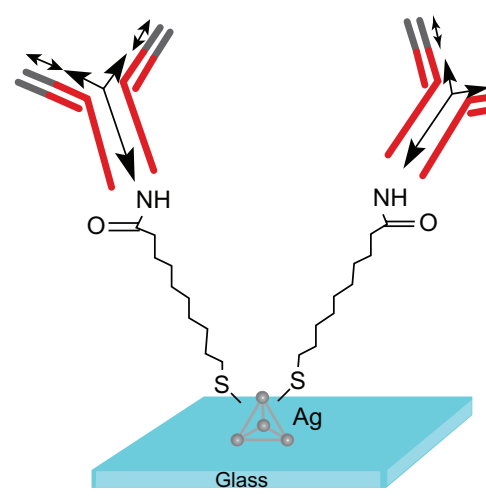

$\mathbf{E}$
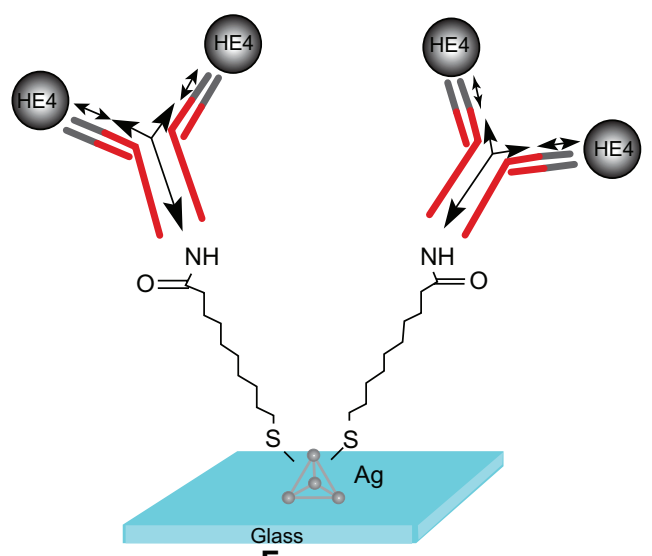

F

Figure 2 Design of the localized surface plasmon resonance biosensor for HE4 detection using a direct assay format. (A) Glass substrate, (B) silver nanoparticles synthesized through NSL technology, (C) A self-assembled monolayer layer formed by incubation in I mM II-mercaptoundecanoic acid, (D) incubation in 75 mM I-ethyl3-(3-dimethylaminopropyl) carbodiimide hydrochloride/I5 mM N-hydroxysuccinimide, (E) anti-HE4 antibody (IO $\mu \mathrm{g} / \mathrm{mL}) \mathrm{covalently}$ attached to the nanoparticles, and (F) different concentrations of the HE4 both in buffer and serum samples reacted with the anti-HE4.

Abbreviation: HE4, human epididymis secretory protein 4.

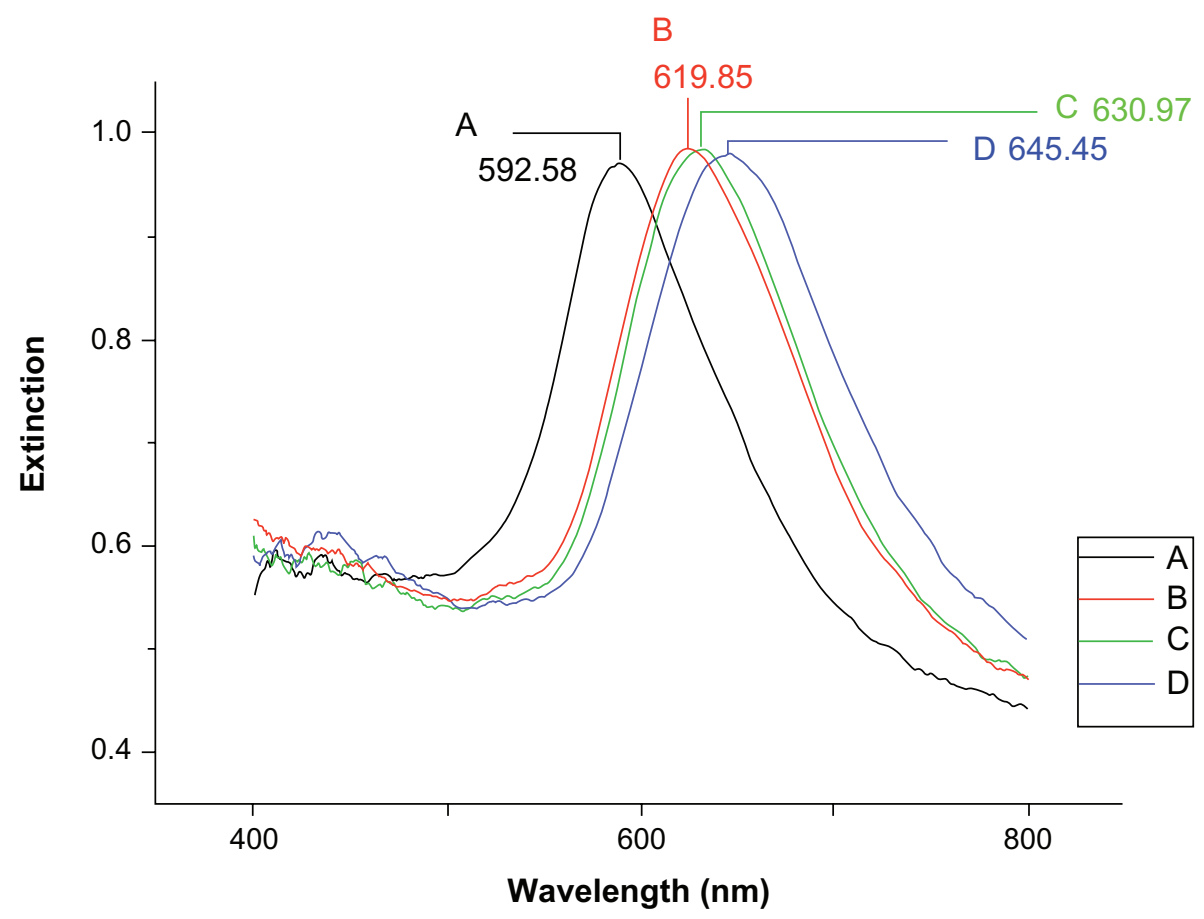

Figure 3 Localized surface plasmon resonance spectra for each step of the detection of 500 pM human epididymis secretory protein 4 . (A) Bare silver nanochip, $\lambda_{\max }=592.58 \mathrm{~nm},(\mathbf{B})$ I mM I I-mercaptoundecanoic acid, $\lambda_{\max }=619.85 \mathrm{~nm}$, (C) functionalized biosensor with $10 \mu \mathrm{gg} / \mathrm{mL}$ antibody, $\lambda_{\max }=630.97 \mathrm{~nm}$, and (D) $500 \mathrm{pM} \mathrm{HE} 4$, $\lambda_{\text {max }}=645.45 \mathrm{~nm}$.

Note: All spectra were collected at room temperature in air.

Abbreviation: HE4, human epididymis secretory protein 4. 
According to Mie theory, the peak LSPR position is related to the refractive index around the particle. Therefore, the change in the local refractive index that accompanies the molecular binding can be sensed by the nanoparticles, and the quantitative detection of targets can be achieved by monitoring the $\Delta \lambda_{\max }$ when the analytes are bound to the nanoparticles. ${ }^{10}$

\section{Calibration curve}

Figure 4 shows a calibration curve of the nanosensor constructed by measuring the LSPR wavelength shifts after exposure of the anti-HE4 attachment surface to the HE4 standard solutions of concentrations ranging from $1 \mathrm{pM}$ to $0.1 \mu \mathrm{M}$ under optimal conditions. As seen from the data, the LSPR $\lambda_{\text {max }}$ values increased stepwise with increasing HE4 concentrations. Like many immunoassays, the curve is sigmoid rather than linear. ${ }^{25}$ In addition, a good linear relationship between the LSPR shifts and the logarithm of the HE4 concentration could be fitted to the experimental points from $10 \mathrm{pM}$ to $10,000 \mathrm{pM}$ (inset of Figure 4). The linear regression equation was LSPR $(\mathrm{nm})=3.72 \times \log$ [HE4] $(\mathrm{M})+47.37$, with a linear correlation coefficient (R) of 0.997 .

This linear range is broader than that of the commercial HE4
ELISA kit (15 pM to $900 \mathrm{pM})$, indicating that it is capable of testing the samples without predilution which could not be confirmed through a routine ELISA. Given that the noise level is defined as the standard deviation of the blank $(n=12)$, the limit of detection, defined as the analyte concentration corresponding to a signal-to-noise ratio of three (about $5 \mathrm{~nm}$ ), was estimated to be $4 \mathrm{pM}$. This limit of detection was more than sufficient for analysis of HE4 in serum where normal values of HE4 are considered to be less than $150 \mathrm{pM} .{ }^{26}$ This detection limit is a little better than that obtained using the ELISA method for HE4 (15 pM). Therefore, the LSPR biosensor had good analytical performance for the detection of HE4, and was comparable with ELISA.

\section{Selectivity, precision, regeneration, and stability tests}

Several control experiments were designed to ensure that the results of Figure 4 were not disturbed by nonspecific adsorption. All the experiments were implemented in triplicate. Squamous cell carcinoma (SCC) antigen, another tumor marker and bovine serum albumin, the most abundant protein component in blood serum were chosen as interferences. The functionalized biosensor was incubated

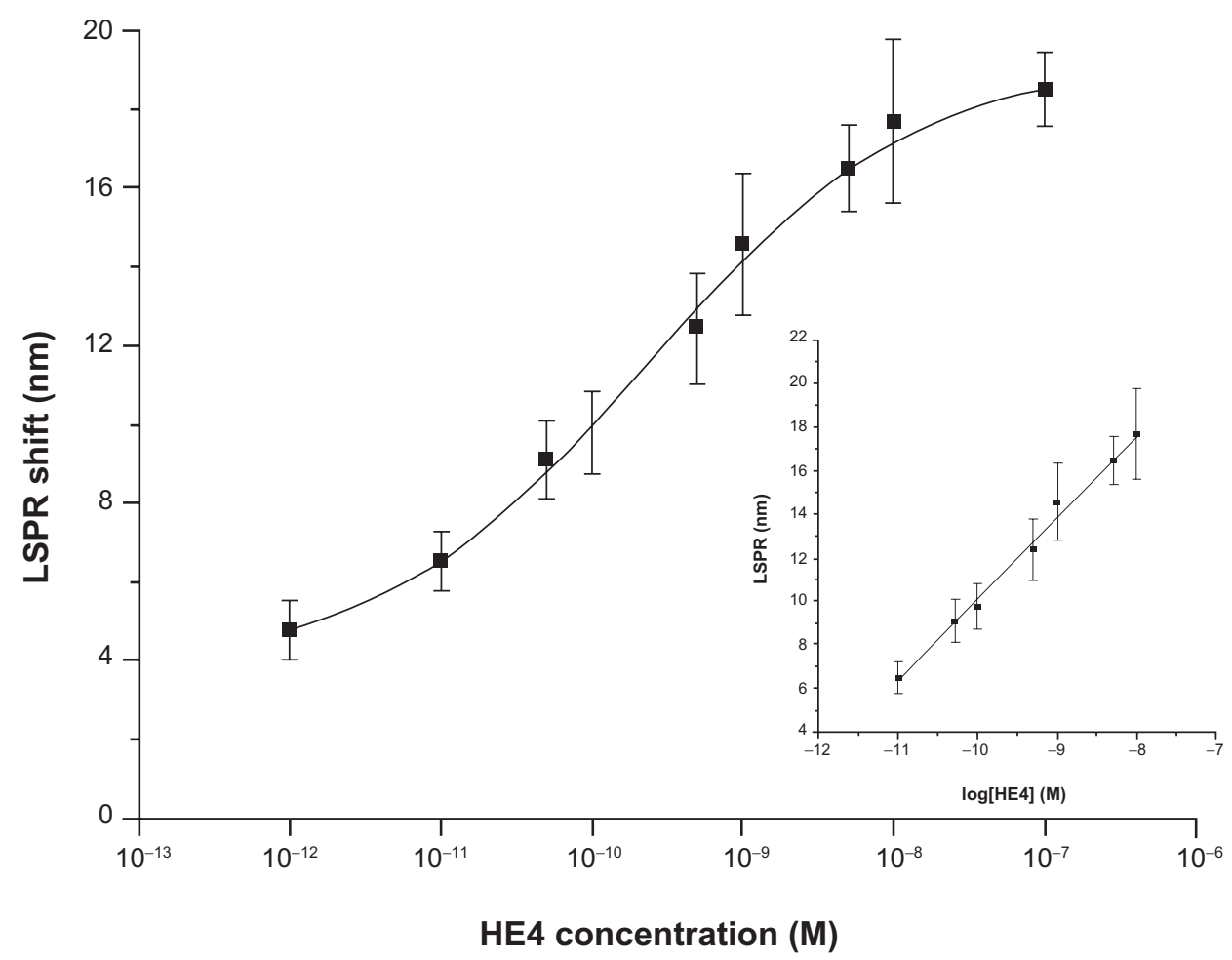

Figure $4 \mathrm{~A}$ semilogarithmic curve of localized surface plasmon resonance shift versus the logarithm of HE4 concentration.

Note: The inset shows the linear relationship between the localized surface plasmon resonance shift and the logarithm of HE4 concentration in the concentration range from 10 pM to 10000 pM.

Abbreviations: HE4, human epididymis secretory protein 4; LSPR, localized surface plasmon resonance. 
with a solution of $500 \mathrm{pM}$ SCC or bovine serum albumin. The same concentration of HE4 was also introduced to the nanosensor surface in the absence of anti-HE4. After phosphate-buffered solution washing, the LSPR shift value was measured. No discernible LSPR shift was observed. Thus, the nonspecific binding of protein molecules on the self-assembled monolayer-covered nanosensor was found to be relatively low. The results indicated that the selectivity of the LSPR biosensor based on the highly specific antigenantibody reaction and surface passivation with ethanolamine was excellent.

The precision within and between batches is an important factor in the practical application of the biosensor. ${ }^{25}$ The intrarun precision was tested at two HE4 concentration levels (500 pM and $5000 \mathrm{pM}$ ) using the LSPR biosensors of the same batch for five continuous measurements within one day. The interrun precision was tested similarly with five biosensors, which were selected randomly from five batches. The results in Table 1 suggest that the nanobiosensor showed acceptable precision and reproducibility.

Following completion of the antigen-antibody reaction, the nanochips were regenerated via exposure to $8 \mathrm{M}$ urea solution, and then washed with ultrapure water. The reproducibility of responses from the identical biosensor and from different biosensors was evaluated by measuring $500 \mathrm{pM}$ standard HE4 solution, and the coefficient of variation for the LSPR shift was obtained. After performing the corresponding HE4 incubation with the freshly regenerated biosensor four times, a coefficient of variation of $14.7 \%$ was obtained, as shown in Table 2. This value indicates that the anti-HE4modified sensor can provide reliable detection of HE4. The biosensor stored in the refrigerator at $4{ }^{\circ} \mathrm{C}$ was regenerated and observed every week for one month. After one, 2, 3, and 4 weeks, the extent of response for 500 pM HE4 dropped by $2.30 \%, 7.21 \%, 9.66 \%$, and $17.09 \%$, respectively, compared with the initial signal. This drop in response seems to be related to gradual deactivation of the anti-HE4 antibody. The LSPR biosensor retained $80 \%$ of its initial responsiveness after regeneration seven times. The silver surface is susceptible to oxidative damage, which could directly affect

Table I Precision tests of LSPR biosensor for HE4

\begin{tabular}{lllll}
\hline $\begin{array}{l}\text { HE4 concentration } \\
(\mathrm{pM})\end{array}$ & $\begin{array}{l}\text { Intrarun } \\
(\mathbf{n}=\mathbf{5}, \mathbf{n m})\end{array}$ & $\mathbf{C V}(\%)$ & $\begin{array}{l}\text { Interrun } \\
(\mathbf{n}=\mathbf{5}, \mathbf{n m})\end{array}$ & $\mathbf{C V}(\%)$ \\
\hline 500 & $12.16 \pm 1.13$ & 9.29 & $12.63 \pm 0.90$ & 7.13 \\
5000 & $15.61 \pm 1.11$ & 7.11 & $16.18 \pm 1.16$ & 7.17 \\
\hline
\end{tabular}

Abbreviations: LSPR, localized surface plasmon resonance; HE4, human epididymis secretory protein 4 ; CV, coefficient of variation.
Table 2 Regeneration and reusability of the LSPR biosensor

\begin{tabular}{llllllll}
\hline $\begin{array}{l}\text { Biosensor } \\
\text { code }\end{array}$ & \multicolumn{2}{l}{ LSPR shift (nm) } & & $\begin{array}{l}\text { Mean } \\
(\mathbf{n m})\end{array}$ & SD & CV (\%) \\
\hline $\mathrm{I}^{\mathrm{a}}$ & 13.86 & 12.44 & 14.44 & 10.23 & 12.74 & $\mathrm{I} .87$ & 14.70 \\
$2^{\mathrm{b}}$ & 13.54 & 12.52 & $\mid 2.86$ & 11.49 & 12.60 & 0.85 & 8.24 \\
\hline
\end{tabular}

Notes: Each LSPR shift obtained corresponds to a measurement cycle when the tested biosensor was freshly regenerated followed by the LSPR shift measurement procedures. Concentration of HE4 was 500 pM. aMeasurement with a freshly prepared anti-HE4-modified biosensor; ${ }^{b}$ measurement with the same anti-HE4modified biosensor through 4 weeks of use.

Abbreviations: LSPR, localized surface plasmon resonance; SD, standard deviation; $\mathrm{CV}$, coefficient of variation.

the stability of the LSPR sensor. However, the nanochips were stable in air after chemical modification through to the end of the 4-week study. These results indicate good reusability and stability for the LSPR biosensor.

\section{Comparison of results from the LSPR system and ELISA}

This study investigated the availability of a domestic system for detecting HE4 in serum. A series of ten human serum samples, five from the ovarian cancer group and five from the control group, were screened for serum HE4 levels using the proposed LSPR sensor and an ELISA kit. According to the literature, the cutoff value for HE4 with $98 \%$ specificity is $150 \mathrm{pM} .{ }^{26}$ Therefore, a sample was defined positive when its concentration was more than $150 \mathrm{pM}$. The five ovarian cancer samples with red shifts ranging from $11 \mathrm{~nm}$ to $17 \mathrm{~nm}$ were shown to be positive. Meanwhile, red shifts less than $10.5 \mathrm{~nm}$ were observed in the negative controls. Thus, consistent results were achieved between the LSPR and ELISA methods in terms of semiquantitative analysis. Based on a $t$-test, differences in HE4 levels between the ovarian cancer group and the control group detected were statistically significant $(P<0.05)$ using both LSPR and ELISA. Furthermore, the LSPR sensor could specifically distinguish between ovarian cancer and the negative controls without the need for labeling in response to HE4 binding in the sera tested. The LSPR biosensor clearly has good specificity that cannot be disrupted by other proteins or components in serum.

The concentration of the ten samples was found to be within the range of 11.39-911.16 pM, which was also within the linear range of the LSPR sensor. A comparison between the two methods of analysis was also done using the regression line method. ${ }^{27}$ The results are shown in Figure 5. The analytical curve was calibrated using the correlation equation, ie, $y=83.88 x-677.07$, with a correlation coefficient of 0.926 . The results indicate that the LSPR biosensor could serve as a good alternative to the laborious 


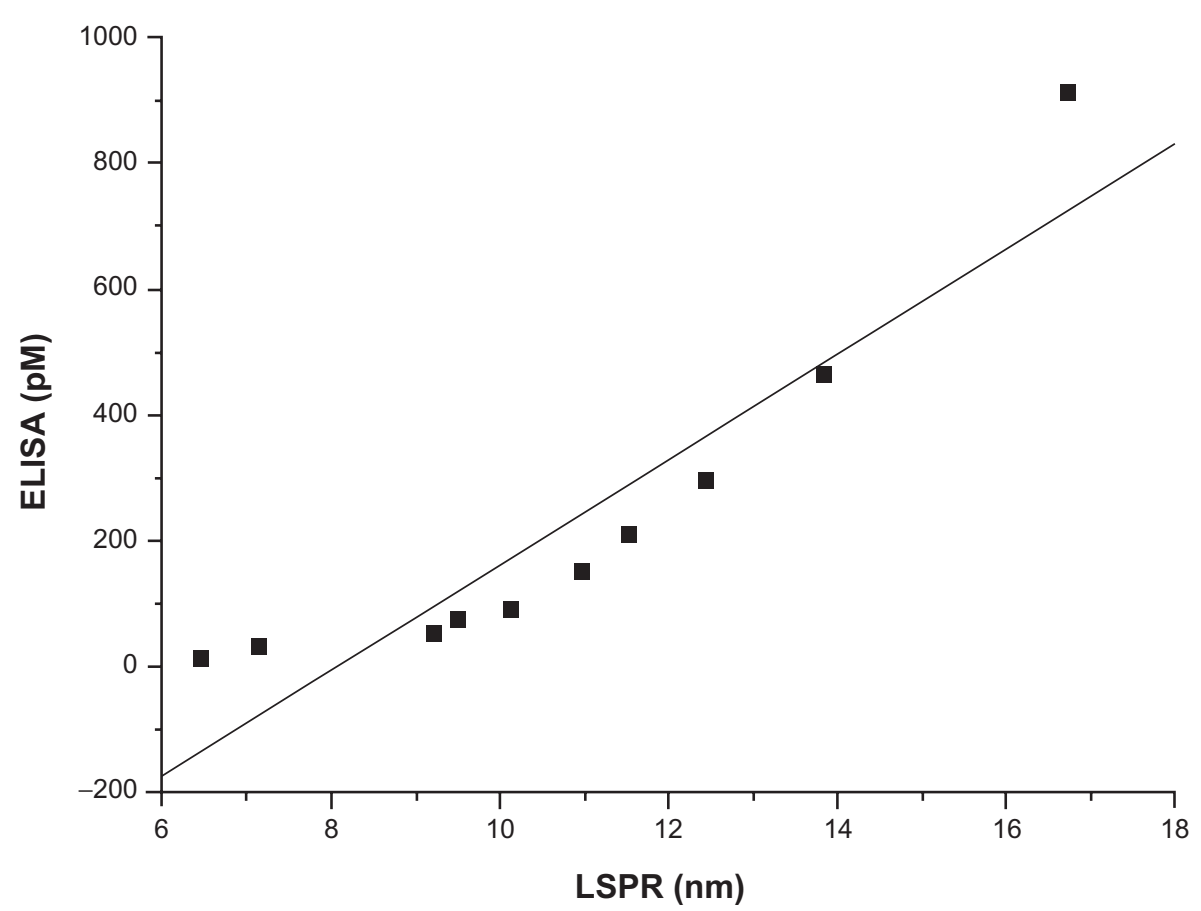

Figure $\mathbf{5}$ Method comparison of ten samples detected by localized surface plasmon resonance and enzyme-linked immunosorbent assay. Abbreviations: LSPR, localized surface plasmon resonance; ELISA, enzyme-linked immunosorbent assay.

and time-consuming ELISA method for direct detection of HE4. Moreover, the technological barrier regarding transfer of bench research to clinical application was overcome to a certain extent. In terms of product commercialization, serum HE4 can be detected in real time using an antibody-coated LSPR sensor within 40 minutes without predilution, thereby reducing the chance of potential procedural errors.

\section{Conclusion}

Ideal biosensors should be rapid, sensitive, specific, label-free, stable, reproducible, cheap, portable, and easy to operate. ${ }^{28}$ The LSPR technique has many of these characteristics, making this method comparable with other immunoassay techniques. The LSPR biosensor has significant advantages in terms of label-free biomarker detection, a rapid test time, and in a direct assay format unlike the traditional immunoassay approaches, such as ELISA. Compared with chemiluminescence analysis and current commercial surface plasmon resonance sensors, the LSPR sensor has outstanding features, including miniaturization, portability, and low cost.

A custom-built LSPR system was used for the first time in medical diagnostics in the field of gynecological oncology. The experiments described in this study demonstrate that a label-free LSPR technique could serve as a very effective alternative to the label-based conventional ELISA method. Furthermore, direct detection of protein targets in human serum, which retains the native specific properties of antibodies or proteins without complicated procedures, makes the LSPR method a very attractive strategy for cancer biomarker studies. Future studies need to be performed to construct a serum calibration curve. A large, randomized, case-controlled clinical study can be used to evaluate the applicability of this biosensor in medical diagnostics for tumors such as ovarian cancer. The LSPR biosensor is anticipated to be a promising platform for point-of-service medical diagnostics and should rival the commercially available instruments.

\section{Acknowledgments}

This study was supported by grants from the National Key Basic Research Program of China (2011CB301800) and the Natural Science Foundation of China (60736037). The authors thank Ting Lai and Wenhao Deng for their kind contribution to this work.

\section{Disclosure}

The authors report no conflicts of interest in this work.

\section{References}

1. Anker JN, Hall WP, Lyandres $\mathrm{O}$, et al. Biosensing with plasmonic nanosensors. Nat Mater. 2008;7:442-453.

2. Nam JM, Thaxton CS, Mirkin CA. Nanoparticle-based bio-bar codes for the ultrasensitive detection of proteins. Science. 2003;301:1884-1886. 
3. Haes AJ, Duyne RP. Preliminary studies and potential applications of localized surface plasmon resonance spectroscopy in medical diagnostics. Expert Rev Mol Diagn. 2004;4:527-537.

4. Haes AJ, Chang L, Klein WL, et al. Detection of a biomarker for Alzheimer's disease from synthetic and clinical samples using a nanoscale optical biosensor. J Am Chem Soc. 2005;127:2264-2271.

5. Sepúlveda B, Angelomé PC, Lechuga LM, et al. LSPR-based biosensors. Nano Today. 2009;4:244-251.

6. Monk DJ, Walt DR. Optical fiber-based biosensors. Anal Bioanal Chem. 2004;379:931-945.

7. Haes AJ, Zou SL, Schatz GC, et al. Nanoscale optical biosensor: short range distance dependence of the localized surface plasmon resonance of noble metal nanoparticles. J Phys Chem B. 2004;108:6961-6968.

8. Yonzon CR, Jeoung E, Zou S, et al. A comparative analysis of localized and propagating surface plasmon resonance sensors: the binding of concanavalin a to a monosaccharide functionalized self-assembled monolayer. J Am Chem Soc. 2004;126:2669-2676.

9. Nath N, Chilkoti A. Label-free biosensing by surface plasmon resonance of nanoparticles on glass: optimization of nanoparticle size. Anal Chem. 2004;76:5370-5378.

10. Nath N, Chilkoti A. A colorimetric gold nanoparticle sensor to interrogate biomolecular interactions in real time on a surface. Anal Chem. 2002;74:504-509.

11. Fujiwara K, Watarai $\mathrm{H}$, Itoh $\mathrm{H}$, et al. Measurement of antibody binding to protein immobilized on gold nanoparticles by localized surface plasmon spectroscopy. Anal Bioanal Chem. 2006;386:639-644.

12. Endo T, Yamamura S, Kerman K, et al. Label-free cell-based assay using localized surface plasmon resonance biosensor. Anal Chim Acta. 2008;614:182-189.

13. Haes AJ, Hall WP, Chang L, et al. A localized surface plasmon resonance biosensor: first steps toward an assay for Alzheimer's disease. Nano Lett. 2004;4:1029-1034.

14. Zhu SL, Li F, Du CL, et al. A localized surface plasmon resonance nanosensor based on rhombic Ag nanoparticle array. Sens Actuators B Chem. 2008;134:193-198.

15. Lai T, Hou QN, Yang H, et al. Clinical application of a novel silver nanoparticles biosensor based on localized surface plasmon resonance for detecting the microalbuminuria. Acta Biochim Biophys Sin (Shanghai). 2010;42:787-792
16. Neesham D. Ovarian cancer screening. Aust Fam Physician. 2007;36: 126-128.

17. Hellström I, Raycraft J, Ledbetter MH, et al. The HE4(WFDC2) protein is a biomarker for ovarian carcinoma. Cancer Res. 2003;63:3695-3700.

18. Montagnana M, Danese E, Giudici S, et al. HE4 in ovarian cancer: from discovery to clinical application. Adv Clin Chem. 2011;55:1-20.

19. Hellstrom I, Heagerty PJ, Swisher EM, et al. Detection of the HE4 protein in urine as a biomarker for ovarian neoplasms. Cancer Lett. 2010;296:43-48.

20. Huhtinen K, Suvitie P, Hiisa J, et al. Serum HE4 concentration differentiates malignant ovarian tumors from ovarian endometriotic cysts. Br J Cancer. 2009;100:1315-1319.

21. Bast RC, Badgwell D, Lu Z, et al. New tumor markers: CA125 and beyond. Int J Gynecol Cancer. 2005;15:274-281.

22. Vaisocherova H, Faca VM, Taylor AD, et al. Comparative study of SPR and ELISA methods based on analysis of CD166/ALCM levels in cancer and control human sera. Biosens Bioelectron. 2009;24: 2143-2148

23. Ma WY, Yao J, Yang H, et al. Effects of vertex truncation of polyhedral nanostructures on localized surface plasmon resonance. Opt Express. 2009; 17:14967-14976.

24. Doron-Mor I, Cohen H, Barkay Z, et al. Sensitivity of transmission surface plasmon resonance (T-SPR) spectroscopy: self-assembled multilayers on evaporated gold island films. Chemistry. 2005;11: 5555-5562.

25. Chalquest RR. Quantitation of indirect sandwich enzyme-linked immunosorbent assay parameters. J Clin Microbiol. 1988;26:147-148.

26. Chang XH, Ye X, Dong L, et al. Human epididymis protein 4 (HE4) as a serum tumor biomarker in patients with ovarian carcinoma. Int J Gynecol Cancer. 2011;21:852-858.

27. Krouwer JS, Tholen DW, Garber CC, et al. EP9-A2: Method Comparison and Bias Estimation Using Patient Samples: Approved Guideline, 2nd ed. Wayne, PA: National Committee for Clinical Laboratory Standards; 2002.

28. Dmitri I, Ihab AH, Plamen A, et al. Biosensors for detection of pathogenic bacteria. Biosens Bioelectron. 1999;14:599-624.
International Journal of Nanomedicine

\section{Publish your work in this journal}

The International Journal of Nanomedicine is an international, peerreviewed journal focusing on the application of nanotechnology in diagnostics, therapeutics, and drug delivery systems throughout the biomedical field. This journal is indexed on PubMed Central, MedLine, CAS, SciSearch $\AA$, Current Contents ${ }^{\circledR} /$ Clinical Medicine,

\section{Dovepress}

Journal Citation Reports/Science Edition, EMBase, Scopus and the Elsevier Bibliographic databases. The manuscript management system is completely online and includes a very quick and fair peer-review system, which is all easy to use. Visit http://www.dovepress.com/ testimonials.php to read real quotes from published authors. 